\section{Patients with chronic obstructive pulmonary disease: management considerations for the dental team}

\author{
J. Devlin ${ }^{1}$
}

\section{VERIFIABLE CPD PAPER}

\section{IN BRIEF}

- Describes some of the medical and dental issues that will impact on the management of patients with chronic obstructive pulmonary disease (COPD) by general dental practitioners for example, candida infection with steroid inhalers and dry mouth symptoms.

- Explains why encouraging smoking cessation is important in preventing COPD.

- Concludes that better periodontal health may result in fewer exacerbations of COPD.

Chronic obstructive pulmonary disease (COPD) affects an estimated 3 million people in the United Kingdom, and is most common among elderly smokers. Patients may present with recurrent coughing of mucoid secretions (chronic bronchitis) or breathlessness caused by destruction of the airways (emphysema). If possible, it is advisable to treat the severely affected patients with them sitting upright in the dental chair as they may find it difficult to breathe when lying in the horizontal position. Periodontal bacteria can be carried into the lung where they can cause respiratory infection; therefore oral hygiene instruction should be emphasised in these patients. The objective of this article is to describe the oral and dental implications of chronic obstructive pulmonary disease. In particular, there have been a number of recent developments in the management of patients with COPD that have direct relevance to the dentist. The drug regime used in the treatment of patients with COPD can have profound implications for clinical dental practice, manifested as dry mouth or oral candidiasis. There is also increasing evidence of a link between COPD and both gastro-oesophageal reflux disease and periodontal disease.

\section{WHAT IS CHRONIC OBSTRUCTIVE PULMONARY DISEASE (COPD)?}

Older patients attending the dental surgery for routine treatment often suffer from respiratory illness. Chronic obstructive pulmonary disease (COPD) is a term that describes several lung diseases including chronic bronchitis and emphysema, which are characterised by reduced expiratory flow. Patients may use either term, but in clinical care this distinction is not a practical one to make as these are now regarded as one condition largely managed in similar ways. Chronic bronchitis is defined as the chronic production of sputum for at least 3 months a year for 2 successive years and emphysema is a condition in which the alveoli of the lungs are damaged and enlarged (Fig. 1). This is often the result of cigarette smoking over many years.

The implication for any restorative dental treatment is that patients with severe COPD often request to be treated sitting upright. The accessory muscles of respiration are more effective with the patient in the upright

Specialist Registrar in Respiratory Medicine, Bristol Royal Infirmary, Upper Maudlin Street, Bristol, BS2 8HW Correspondence to: Joanna Devlin

Email: joannadevlin@doctors.org.uk

\section{Refereed Paper}

Accepted 20 May 2014

DOI: 10.1038/sj.bdj.2014.756

${ }^{\circledR}$ British Dental Journal 2014; 217: 235-237 position. Oxygenation of the blood may be reduced in the supine patient with advanced COPD. In some patients, the chest and neck muscles are used to assist in the respiratory effort so that performing restorative dentistry on them can be difficult. In particular, the water spray and the application of rubber dam can further inhibit their already compromised breathing action.

\section{WHAT CAUSES DYSPNOEA IN EMPHYSEMA?}

The main pathology underlying the breathing difficulty seen in patients with emphysema is the irreversible loss of recoil of the alveoli during expiration (Fig. 2). Thus the patient with severe emphysema has to inspire more quickly on an already inflated lung to allow more time to exhale.

COPD also causes ventricular hypertrophy and pulmonary hypertension. The function of the right ventricle is to deliver deoxygenated blood from the body to the lungs where it is oxygenated and then it returns to the left atrium, and thence to the left ventricle of the heart. The oxygenated blood is pumped by the left ventricle to the body. In a patient unaffected by COPD, the circuit from the heart to the lungs is a low pressure system. The effect of smoking is to cause a reduction in the number and diameter of pulmonary capillaries as they are stretched over the larger alveoli air spaces. ${ }^{1}$ In some patients

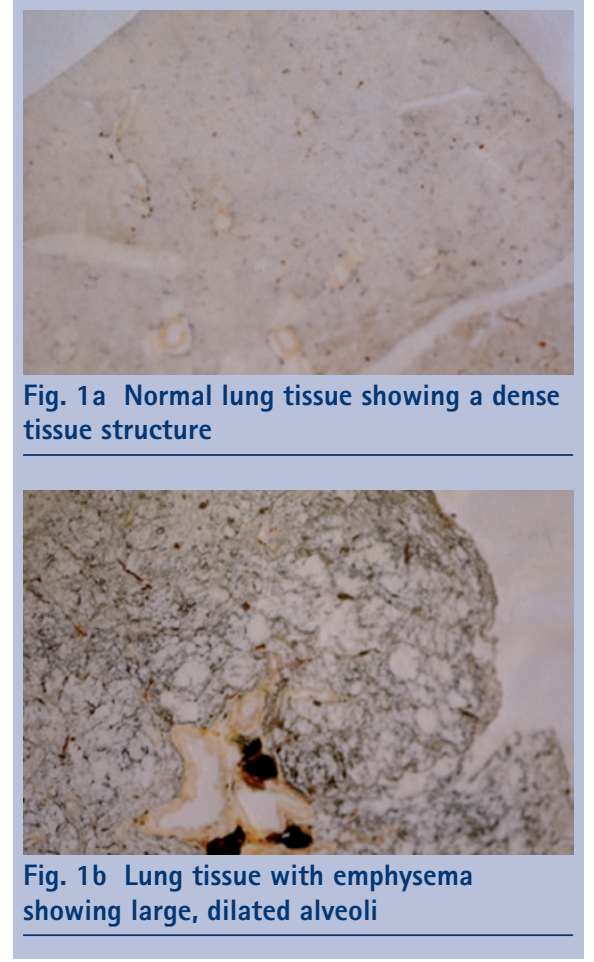

with COPD, the increase in pulmonary vascular resistance causes an increased mean pulmonary arterial pressure greater than $20 \mathrm{~mm} / \mathrm{Hg}$ (which is the definition of pulmonary hypertension). Patients with COPD and severe pulmonary hypertension suffer greater dyspnoea and have a shorter life expectancy. ${ }^{2}$ 
Adrenaline in local anaesthetic can be used with caution in patients with drugcontrolled hypertension; the limit currently advised is two cartridges of 2\% lidocaine with 1 in 80,000 adrenaline (equivalent to a dose of $45 \mu \mathrm{g}$ of adrenaline). ${ }^{3}$

\section{MANAGEMENT OF COPD}

The pharmacological options for treatment are limited, but inhaled short and long acting beta agonists, inhaled steroids and inhaled long acting muscarinic antagonists act to dilate the bronchi and reduce airway inflammation. Short-acting beta-agonists, such as salbutamol, can be taken before dental treatment to aid breathing.

Inhaled corticosteroids may be prescribed by a doctor for those COPD patients with severe and very severe airflow obstruction, where there is an exacerbation or persistent breathlessness. ${ }^{4}$ Inhaled corticosteroids are associated with an increased risk of non-fatal pneumonia and oropharyngeal candidosis (Fig. 3). ${ }^{5,6}$ Elderly people wearing complete dentures and patients who have taken repeated courses of antibiotics are particularly vulnerable to candidal infection, although all patients using a steroid-containing inhaler should be advised to rinse out their mouth having used it. A spacer device used with the inhaler can reduce the deposition of steroid in the mouth and contribute to reducing the risk of candidal infection. Patients having an exacerbation of their COPD may be prescribed oral corticosteroids. This is usually done for short periods to avoid the potential for adrenal atrophy, raised blood glucose levels and osteopenia/osteoporosis that may result in bisphosphonate prescription with the associated risks to oral health of this group of drugs.

The single medical intervention that confers the largest survival benefits for cardiovascular and lung cancer mortality in COPD is that of smoking cessation. As the dentist sees these patients regularly, encouraging patients to give up smoking is an important role. Drugs such as nicotine replacement therapy, varenicline and bupropion may be offered to patients who wish to give up smoking as part of the NHS stop smoking programme. Dry mouth may be a side effect of both drugs requiring the prescription of artificial saliva. COPD can result in mouth breathing, which causes further drying of the gingival tissues around the upper anterior teeth and gingivitis. It is not known whether there is also an increased prevalence of caries among patients with COPD.

\section{THE USE OF EMERGENCY OXYGEN}

In the emergency situation where dental patients with COPD develop hypoxia,

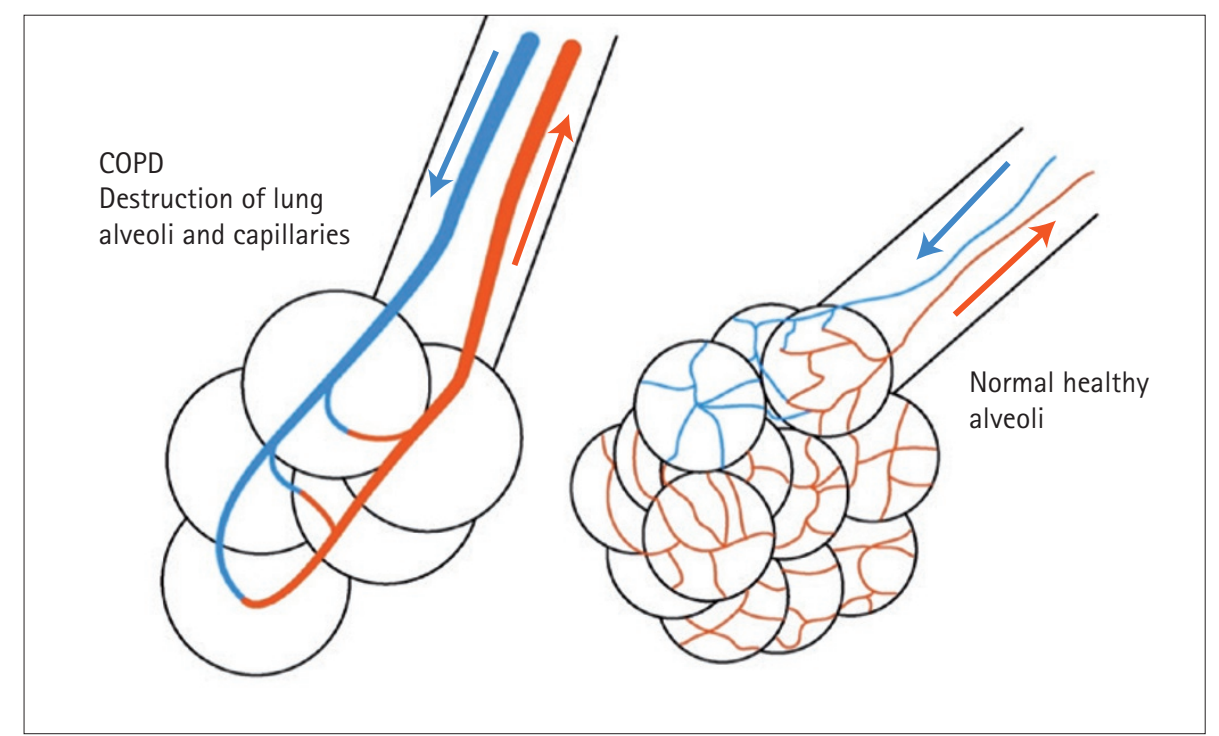

Fig. 2 In emphysema there is destruction of either the respiratory bronchioles or acini or both

they will need to be given oxygen. A small minority of COPD patients are well acclimatised to hypoxia and rely on it to drive respiration. High flow oxygen may therefore have the effect of reducing their respiratory rate due to the loss of their hypoxic drive to respiration, putting the patient at risk of developing hypercapnic respiratory failure. The 2011 British Thoracic Society guidelines on the use of emergency oxygen suggest that all locations where emergency oxygen may be used must have pulse oximetry available. ${ }^{7}$ Patients thought to be at risk of developing hypercapnic respiratory failure should have a target oxygen saturation of 88-92\%, while the suggested target saturation range for most patients is 94-98\%.7 In an emergency, hypoxia kills faster than carbon dioxide related respiratory depression. This means that COPD patients who develop respiratory distress acutely in the dental setting should be treated initially with high flow oxygen. The oxygen flow can be reduced slowly if the respiratory rate drops. The protocol for administration of oxygen, the equipment used and the subsequent discharge of the patient to medical follow-up have been described by Jevon. ${ }^{8}$

\section{PREVENTING EXACERBATIONS OF COPD}

The aspiration of high concentrations of virulent bacteria-contaminated saliva into the lungs may be a cause of pneumonia in debilitated patients. Periodontal disease and COPD are both chronic inflammatory diseases associated with a systemic inflammatory response that can present with acute exacerbations. In both of these conditions cigarette smoking is an important causative factor, leading to infiltration of polymorphonuclear leucocytes such as neutrophils and the release of proteolytic

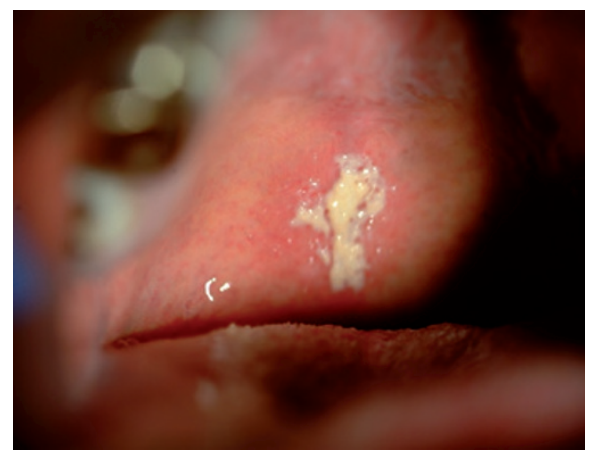

Fig. 3 Overgrowth of Candida albicans on the soft palate of a patient using inhaled corticosteroids

enzymes responsible for tissue damage. In assessing the relationship between COPD and periodontal disease, the role of smoking and age as potential confounding factors must be included in any statistical model to avoid a spurious relationship.

In a paper published very recently, edentulism was associated with a higher frequency of COPD-related hospitalisation and death than those with good periodontal health. ${ }^{9}$ It is hypothesised that those who have lost their teeth through an exaggerated periodontal inflammatory response may also be susceptible to inflammation-induced incidents associated with COPD. High quality evidence is lacking and a causal explanation unproven, but it is assumed that better periodontal health in patients with COPD will result in fewer exacerbations of their disease.

Research has shown that pulmonary rehabilitation programmes for patients with COPD are often poorly attended. ${ }^{10}$ The reasons given by patients for nonattendance at both these programmes and

\section{LOWERING THE BARRIERS TO EFFECTIVE DENTAL CARE FOR PATIENTS WITH COPD}


dental practice appointments will probably be similar. Patients say that a lack of transport is important and those who are socially isolated and living alone are most likely to be affected. Patients who have severe disease have poor mobility, dyspnea and frequent hospitalisation, all of which make regular dental attendance problematic.

\section{CAN GOOD PERIODONTAL HEALTH REDUCE THE FREQUENCY OF INFECTIVE EXACERBATIONS OF COPD?}

The mouth is a reservoir for potential respiratory pathogens; methicillinresistant staphlococcus aureus (MRSA) and Pseudomonas aeruginosa have been found to colonise the oral cavity of a proportion of patients with COPD. ${ }^{11,12}$ Colonisation is thought to emerge as a result of antibiotic suppression of the normal oral bacteria and from the reduced salivary secretion often found in debilitated COPD patients. Bacterial pneumonia can subsequently result from aspiration of these organisms. When the sputum of patients with an exacerbation of chronic bronchitis was analysed, there were raised antibody levels against Fusobacterium nucleatum and Prevotella intermedia..$^{13}$ These are anaerobic organisms that have been implicated in chronic periodontitis.

In a prospective, controlled trial, Kucukcoskun et al. ${ }^{14}$ found that periodontal treatment significantly reduced the frequency of COPD exacerbations. Although median exacerbations increased from two to three per year in the control group, which received no intervention, they declined from three to two per year in the test group, and this was thought to be due to reduced pathogen colonisation on the surfaces of teeth. ${ }^{11}$ A recent joint position paper by the European Federation of Periodontology and American Academy of Periodontology found that there was no firm evidence of a causal link between COPD and periodontal disease. However, they acknowledged that the evidence for an association between aspiration of plaque organisms and pneumonia is much stronger. ${ }^{15}$ It is important that both doctors and dentists continue to educate their COPD-suffering patients of the benefits of good oral and denture hygiene in preventing exacerbations.

\section{SUMMARY}

Chronic obstructive pulmonary disease causes patients to suffer from dyspnoea, and this is likely to compromise any routine dental care. In understanding the pathophysiology, routine pharmacological management and its side effects, as well as the importance of good periodontal health in preventing both infective and noninfective COPD exacerbations, the physician and dentist can work together to provide a holistic approach to improving their patients morbidity and mortality.

1. Yamato H, Sun J P, Churg A, Wright J L. Cigarette smoke-induced emphysema in guinea pigs is associated with diffusely decreased capillary density and capillary narrowing. Lab Invest 1996; 75: 211-219.

2. Weitzenblum E, Hirth C, Ducolone A, Mirhom R, Rasaholinjanahary J, Ehrhard M. Prognostic value of pulmonary artery pressure in chronic obstructive pulmonary disease. Thorax 1981; 36: 752-758.

3. Brown R S, Rhodus N L. Adrenaline and local anaesthesia revisited. Oral Surg Oral Med Oral Pathol Oral Radiol Endod 2005; 100: 401-408.

4. National Institute for Health and Care Excellence Chronic obstructive pulmonary disease (updated) CG101. London: NICE, 2010. Online guidelines available at http://guidance.nice.org.uk/CG101 (accessed June 2014).

5. Suissa S, Patenaude V, Lapi F, Ernst P. Inhaled corticosteroids in COPD and the risk of serious pneumonia. Thorax 2013; 68: 1029-1036.

6. Janson C, Larsson K, Lisspers K H et al. Pneumonia and pneumonia related mortality in patients with COPD treated with fixed combinations of inhaled corticosteroid and long acting $\beta 2$ agonist: observational matched cohort study (PATHOS). BMJ 2013; 346: f3306.

7. British Thoracic Society. BTS guidelines for emergency oxygen use in adult patients. BTS, 2008.

8. Jevon P. Emergency oxygen therapy in the dental practice: administration and management. Br Dent J 2014; 216: 113-115.

9. Barros S P, Suruki R, Loewy Z G, Beck J D, Offenbacher $S$. A cohort study of the impact of tooth loss and periodontal disease on respiratory events among COPD subjects: modulatory role of systemic biomarkers of inflammation. PLoS One 2013; 8: e68592.

10. Hayton C, Clark A, Olive S et al. Barriers to pulmonary rehabilitation: characteristics that predict patient attendance and adherence. Respir Med 2013; 107: 401-407.

11. Scannapieco FA, Stewart E M, Mylotte J. Colonization of dental plaque by respiratory pathogens in medical intensive care patients. Crit Care Med 1992; 20: 740-745.

12. Johnson W G, Pierce A K, Sandford A K. Changing pharyngeal bacterial flora of hospitalized patients. Ann Int Med 1972; 77: 701-706.

13. Brook I, Frazier H E. Immune response to Fusobacterium nucleatum and Prevotella intermedia in the sputum of patients with acute exacerbations of chronic bronchitis. Chest 2003; 124: 832-833.

14. Kucukcoskun M, Baser U, Oztekin G, Kiyan E, Yalcin F. Initial periodontal treatment for prevention of chronic obstructive pulmonary disease exacerbations. J Periodontol 2013; 84: 863-870.

15. Linden $G J$, Herzberg M C, Working group 4 of the joint EFP/AAP workshop. Periodontitis and systemic diseases: a record of discussions of working group 4 of the Joint EFP/AAP Workshop on Periodontitis and Systemic Diseases. J Periodontol 2013; 84 (Suppl 4): S20-23. 